\title{
Coagulation/fibrinolysis and circulating tumor cells in patients with advanced breast cancer
}

\author{
Luc Y. Dirix ${ }^{1}$ - Steffi Oeyen ${ }^{1} \cdot$ Andy Buys $^{1} \cdot$ Vincent Liégois $^{1} \cdot$ Annemie Prové $^{1} \cdot$ Tom Van De Mooter $^{1}$. \\ Steven Van Laere ${ }^{1} \cdot$ Peter B. Vermeulen ${ }^{1}$
}

Received: 8 October 2021 / Accepted: 3 December 2021 / Published online: 8 February 2022

(c) The Author(s) 2022

\begin{abstract}
Purpose To evaluate the relationship between circulating tumor cells (CTCs) and standard coagulation tests in both a discovery and a validation cohort of patients with advanced breast cancer.

Methods In a retrospective $(n=77)$ and a prospective $(n=92)$ study of patients with progressive advanced breast cancer, CTC count, platelet number, fibrinogen level, D-dimers, prothrombin time, and activated partial thromboplastin time were measured. The association between these coagulation studies and CTC count was analyzed. The impact of these measurements on overall survival (OS) was assessed.

Results In both cohorts, results were similar; absolute CTC count was significantly associated to D-dimer level and inversely with platelet count. In the prospective cohort, quantification of tumor-derived extracellular vesicles (tdEVs) was associated with CTC count, and with coagulation abnormalities (low platelet count and increased D-dimers). tdEVs did not add to CTC count in predicting changes in platelets or D-dimers. In multivariate analysis only CTC $\geq 5$ CTC/7.5 mL, ER status, HER2 status and lines of chemotherapy were associated with OS. In patients with terminally metastatic breast cancer, very high CTC counts are prevalent.

Conclusion A significant association exists between increasing CTC number and increased D-dimers and decreased platelet counts, suggesting a potential role for CTCs as a direct contributor of intravascular coagulation activation. In patients with advanced and progressive breast cancer, abnormalities in routine coagulation tests is the rule. In patients with terminally advanced breast cancer a "leukemic" phase with high CTC count is prevalent.
\end{abstract}

Keywords Advanced breast cancer · Circulating tumor cells · D-dimers · Platelets · Diffuse intravascular coagulation · Tumor derived extracellular vesicles

\section{Introduction}

Coagulation activation is detectable in plasma in the majority of patients with localized and/or advanced breast cancer [1-4]. Its biological origin and eventual clinical significance are less clear and might be underestimated. A relationship between markers of coagulation activation and prognosis has been established [5-7]. This activation might be considered solely as a bystander phenomenon of tumor growth, and eventually serve as a surrogate for disease burden, c.q. disease kinetics. There are however preclinical and clinical

Luc Y. Dirix

luc.dirix@gza.be

1 GZA Sint Augustinus, Oosterveldlaan 24, Wilrijk, 2610 Antwerp, Belgium arguments to consider this activation as an active contributor to tumor dissemination and growth. The role of coagulation activation in stromal remodeling is well established, e.g. the contribution of platelets in tumor angiogenesis as a major source of circulating vascular endothelial growth factor (VEGF) [8-13]. In addition, active coagulation is a prerequisite for successful tumor cell extravasation in numerous preclinical model systems and seems critical for the successful conclusion of the metastatic cascade [14, 15]. Interactions between circulating tumor cells (CTC), platelets and neutrophils including the neutrophil extracellular traps (NETs) are contributing to CTC survival and dissemination [16].

Markers of active coagulation in plasma of patients with cancer, including patients with metastatic breast cancer (MBC), enables the identification of those patients at increased risk for venous thromboembolic events (VTE) 
[17]. Patients with MBC and suffering from a VTE-event have a worse outcome [18]. Finally, in patients with advanced disease, a hypercoagulable state can develop into life threatening diffuse intravascular coagulation (DIC), microangiopathic thrombosis, hemolytic anemia and a combination thereof [19].

Different mechanisms have been proposed to explain this hypercoagulable state. We have previously demonstrated that venous effluent levels of plasma D-dimers in patients with localized colorectal cancer are significantly increased in comparison with arterial levels [20]. This suggests a substantial contribution of local intratumoral coagulation activation to the changes as measured in blood, suggestive of an overflow phenomenon. However, intravascular activation of the coagulation/fibrinolysis might also contribute to the overall result. The presence of tissue factor (TF) expressing circulating tumor cells (CTC) and tdEVs in the circulation is considered as one of the most important contributors to this prothrombotic activity [21].

In this study we have investigated the association between circulating tumor cells and markers of active coagulation/fibrinolysis in two cohorts of patients with MBC. The first cohort consists of patients that have participated in prior trials on CTCs in MBC and for whom, for whatever reason, coagulation tests were performed. We consider this first data set as a discovery cohort. The second cohort consists of patients with MBC that participated in the $\mathrm{P} 1133$ prospective study on different quantification methods for CTC enumeration.

\section{Methods}

\section{Patients}

\section{Cohort 1}

Peripheral blood samples were collected from 77 patients with $\mathrm{MBC}$ as part of different clinical studies exploring the prognostic role of CTC enumeration in MBC at the SintAugustinus Cancer Center (Antwerp, Belgium) between Jan 2001 until June 2016. The original study results have been reported in detail elsewhere [22-25]. The patients in the current analysis have been included provided coagulation tests were available within a window of 10 days from the date of the CTC enumeration. Patients were excluded if they were treated with any type of anticoagulative or anti-platelet drug or if systemic anticancer treatment was switched between CTC and coagulation sampling moments. Patients with an active or past thromboembolic event were also excluded.

\section{Cohort 2}

A second cohort of patients with MBC was enrolled in a prospective clinical trial at the Sint-Augustinus Cancer Center (Antwerp, Belgium) between February 2019 and December 2020. Follow-up was captured until end March 2021. This study entitled "P1133: CTC enumeration using Rarecyte and CellSearch systems: a comparative study" had as primary endpoint the comparison between CTC numeration by the CellSearch and Rarecyte platforms. All patients had either a de novo diagnosis of metastatic disease or evidence of disease progression. Patients on anticoagulation therapy or with a known other malignancy within the last 5 years were excluded. This study was approved by the GzA Ethical committee. Written informed consent was obtained from all individual participants.

\section{Blood sampling}

Peripheral venous blood was collected using CellSave preservative (Menarini, Italy) and citrate vacutainer tubes (BD Biosciences, NJ USA) for CellSearch ${ }^{\circledR}$ (Menarini, Italy) circulating tumor cell (CTC) analysis and platelet poor plasma preparation, respectively. Blood was collected prior to treatment for disease progression. CellSave whole blood samples were stored at room temperature until analysis.

\section{CTC enumeration}

CellSearch ${ }^{\circledR}$ circulating tumor cell (CTC) analysis: CellSave blood samples $(7.5 \mathrm{~mL})$ were processed by the CellSearch ${ }^{\circledR}$ system in the Translational Cancer Research Unit (TCRU) as described elsewhere [26]. Briefly, CTCs are immunomagnetically separated from other blood components by EpCAM (epithelial cell adhesion molecule) antibodyconjugated beads and then stained for cytokeratins (CKs 8, 18 and 19), DAPI (4',6-diamidino-2-phenylindole) and CD45 in a fluorescent-based approach. CTCs are defined as $\mathrm{CK}+\mathrm{DAPI}+\mathrm{CD} 45-$ cells over $4 \mu \mathrm{m}$ in diameter.

\section{Accept}

Archived immunofluorescence images were retrospectively reanalyzed by means of the open source image analysis tool for Automated CTCs Classification, Enumeration and Phenotyping (ACCEPT) (https://github.com/LeonieZ/ ACCEPT). Upon downloading the images into ACCEPT, the algorithm automatically provides phenotypic measurements for all detected events (larger than four pixels) per fluorescence channel. Running validated user-defined gating settings, the software was able to automatically detect and enumerate CTCs and tdEVs, and store the phenotypic 
characteristics of these EpCAM + entities in a ready-to-use database [26].

\section{Statistics}

Statistical analysis GraphPad Prism software version 9.2.0 (CA, USA) was used for primary statistical analysis and graph preparation.

Continuous variables are describes by median and range.

Normality of data was confirmed by D'Agostino-Pearson normality testing for the transformed D-dim, tdEVs and CTC results.

Overall survival was measured as days from study entry till death or censored at last follow-up.

Median OS was estimated by Kaplan-Meier survival curves. Survival curves were compared with a logrank test.

\section{Results}

\section{Retrospective cohort $(n=77)$}

From our CTC database with 839 patients with MBC and at least one CTC count, 77 (9.1\%) patients could be identified in accordance with the limited in- and exclusion criteria. Clinical details are summarized in Table 1.

Table 1 Retrospective patients cohort $1(n=77)$

\begin{tabular}{ll}
\hline Age (median, range) & 63 years (33-91) \\
Pathology & \\
IDA & $55(71.5 \%)$ \\
ILA & $22(28.5 \%)$ \\
ER+/HER2- & $42(54 \%)$ \\
ER-/HER2- & $13(17 \%)$ \\
ER-/HER2+ & $16(21 \%)$ \\
ER+/HER2+ & $6(8 \%)$ \\
Visceral disease & $47(61 \%)$ \\
Non-visceral disease & $30(39 \%)$ \\
Bone only & $21(27 \%)$ \\
Initial presentation with metastasis & $38(49 \%)$ \\
On adjuvant endocrine therapy & $20 / 38$ \\
No prior chemotherapy & $40(52 \%)$ \\
Prior chemotherapy & $37(48 \%)$ \\
1 line & 15 \\
2 lines & 11 \\
3 lines & 7 \\
4 lines & 2 \\
5 lines & 2 \\
Prior trastuzumab & 12 \\
\hline
\end{tabular}

IDA infiltrating ductal adenocarcinoma, ILA infiltrating lobular carcinoma, $E R$ estrogen receptor, HER2 human epidermal growth factor receptor 2
Median age was 63 years (range 33-91). At the time of this analysis, all 77 patients have died from MBC. Survival is counted in elapsed days since the blood sample collection for CTC enumeration. Median overall survival is 178 days (15-3120). Although heterogeneous, this is overall a cohort with poor prognosis. Twenty five (32.5\%) patients had died within three months of study entry.

Median CTC count was $53 / 7.5 \mathrm{~mL}$ (range $0-100,000 / 7.5 \mathrm{~mL}$ ). Only 14 of the 77 patients (18\%) had a CTC count lower than $5 \mathrm{CTC} / 7.5 \mathrm{~mL}$. A histogram of the CTC count distribution is shown in Fig. 1.

All 77 patient had at least one routine coagulation test result outside the limits of normal.

In total 13 patients (16.8\%) were diagnosed with abnormalities compatible with overt DIC.

Only 7 and 5 patients had respectively an increased activated partial thromboplastin time (aPTT) or prothrombin time (PTT). No significant association between aPTT or PTT with either CTC, platelet count or D-dimers could be identified.

\section{Correlation between CTC and OS}

The prognostic significance for overall survival of CTC count at time of progression (<or $\geq 5 \mathrm{CTC} / 7.5 \mathrm{~mL}$ ) was confirmed (Fig. 1). Median OS was respectively 150 days versus 1155 days with a logrank HR of $2.826(p<0.0001)$ (Fig. 2A).

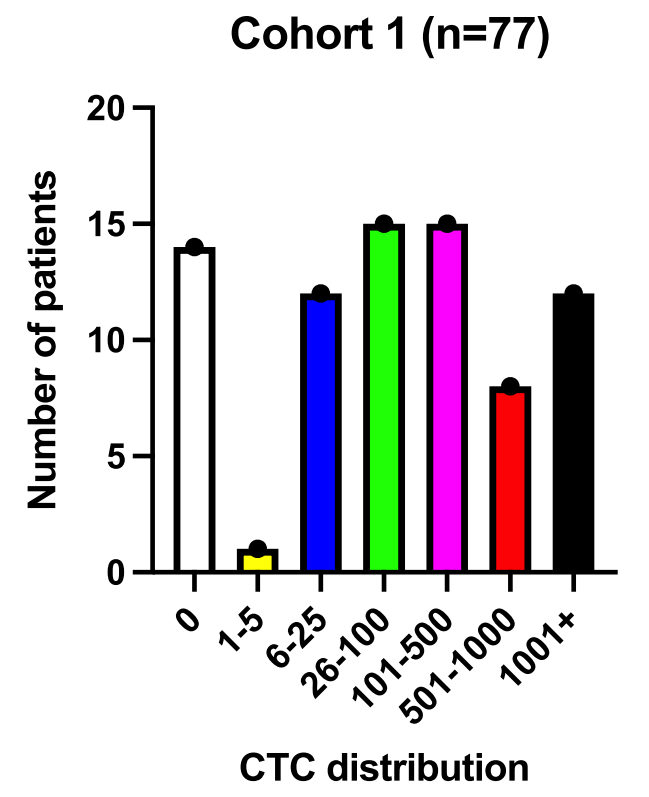

Fig. 1 CTC count distribution cohort 1 
Fig. 2 A Overall SURVIVAL (days) in patients with MBC for those with $<5$ CTCs per $7.5 \mathrm{~mL}$ of whole blood and those in the group with $\geq 5$ CTCs in $7.5 \mathrm{~mL}$ of whole blood $(n=77)$. (log rank HR 2.826, $p<0.0001)$. B1 Relationship between the ln D-dimers (ng/ $\mathrm{mL})$ versus OS (days) $\left(R^{2}=0.465, p<0.0001\right)$. B2 Overall Survival (days) in Patients with MBC for those with $<$ or $>$ median D-dimer $(n=75)$. (log rank HR 2.586, $p<0.0001)$. C Relationship between $\ln$ CTC $(x / 7.5 \mathrm{~mL})$ and $\mathrm{ln}$ D-dimers $(\mathrm{ng} / \mathrm{mL})\left(R^{2}=0.3215, p<0.0001\right)$. D Correlation between platelet count (PLT) $\left(10^{9} / \mathrm{mL}\right)$ and the $\ln$ CTC $(x / 7.5 \mathrm{~mL})\left(p<0.0009, R^{2} 0.167\right)$. E Correlation between platelet count (PLT) $\left(10^{9} / \mathrm{mL}\right)$ and the $\ln \operatorname{Dim}(\mathrm{ng} / \mathrm{mL})(p<0.016)$

\section{Correlation between D-dim and OS}

The levels of D-dimer ranged between 161 and 77,735 ng/ $\mathrm{mL}$ with a median of $2145 \mathrm{ng} / \mathrm{mL}$. Linear regression of the In value of D-dimers with OS was statistically significant $\left(p<0.0001, R^{2}=0.4625\right)$ (Fig. 2B1).

Median OS according to the median D-dim was 93 days versus 450 days with a logrank HR of 2.586 (1.574-4.247) $(p<0.0001)$ (Fig. 2B2).

\section{Correlation between CTC as a continuous variable and D-dim level}

In this cohort a striking linear correlation exist between CTC enumeration and $1 n$ D-dimer level $(p<0.0001$; $\left.R^{2}=0.3215\right)$ (Fig. 2C).

\section{Correlation between CTC and platelet count (PLT)}

The average platelet number varied between 24 and $592 \times 10^{9} / \mathrm{mL}$ with a median value of 206 . The PLT level was correlated with the ln CTC level $(p<0.0009$, $R^{2}=0.167$ ) (Fig. 2D).

\section{Correlation between D-dim and PLT}

Platelet count is negatively correlated with D-dim level $(p<0.016)$ (Fig. 2E).

\section{Univariate and multivariate analysis for OS}

In the univariate analysis, presence of visceral disease, number of lines of chemotherapy ( 0 vs 1 vs $2+$ ), $\ln$ CTC, CTC at baseline, CTC $\geq 5$, the ln D-dimers, platelet count and serum LDH were significantly associated with overall survival. ER status, HER2 status, TN disease, age, and fibrinogen level were not.

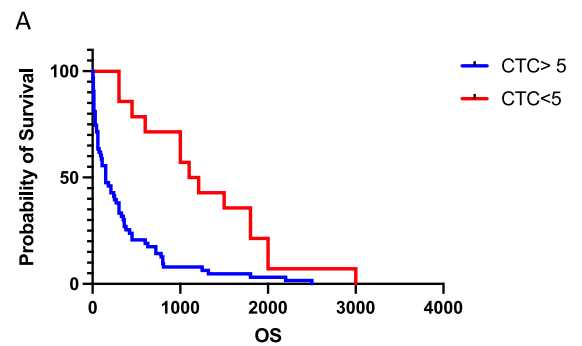

B1

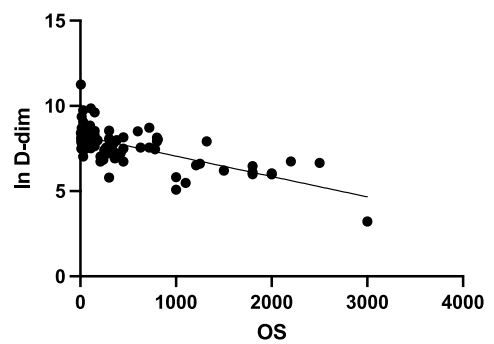

B2

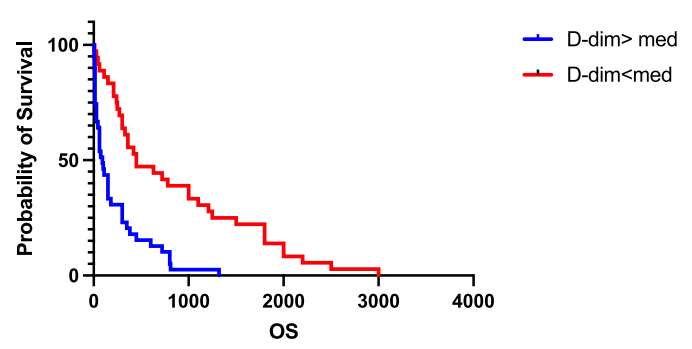

C

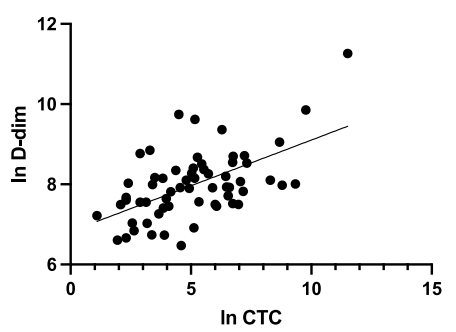

D

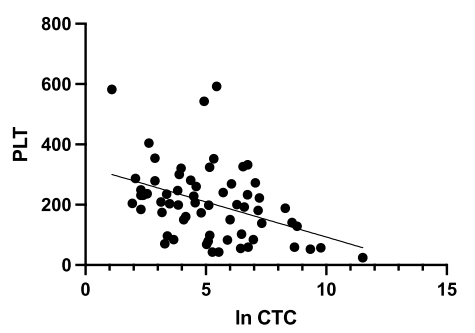

E

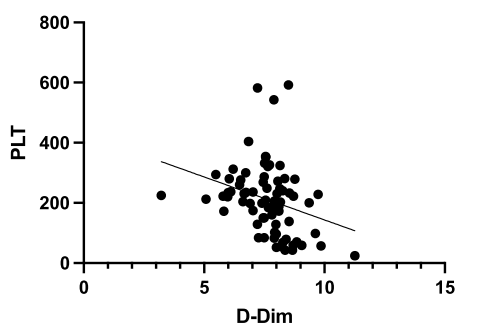


In multivariate analysis only CTC $\geq 5$ at baseline, $\ln$ D-dimers, lines of chemotherapy $0-1$ vs $2+$ ) and presence of visceral disease were associated with OS.

\section{Prospective cohort $(n=92)$}

One hundred and four patients with MBC were prospectively enrolled in a comparative CTC enumeration study called "P1133". As one of the secondary endpoints in that study, coagulation studies (aPTT, PT, Fibrinogen and D-dimers) were systematically performed synchronous with the CTC enumeration sampling. Twelve patients with thromboembolic events and/or any type of anticoagulative therapy were excluded from the current analysis. Clinical details are summarized in Table 2. Median age was 63 years (range 34-97), nearly 4 out 5 patients suffered from ER+/HER2- disease and one in three patients presented ab initio with stage IV disease. Only $17 \%$ had received prior chemotherapy for metastatic disease. Median follow-up was 210 days (range 15-751). Median overall survival has not been reached. The CellSearch ${ }^{\circledR}$ CTC count had a median of 4 tumor cells $/ 7.5 \mathrm{~mL}$ (range 0-2289). This cohort of patients had in general less advanced disease both with regard to extent of disease and number of prior treatment regimens. A histogram of the CTC count distribution is shown in Fig. 3 with only 5 patients had a CTC count in excess of 500 tumor cells $/ 7.5 \mathrm{~mL}$.

Table 2 prospective patients cohort $2(n=92)$

\begin{tabular}{ll}
\hline Age (median, range) & 63 years (34-97) \\
Pathology & $69(75 \%)$ \\
IDA & $23(25 \%)$ \\
ILA & $73(79 \%)$ \\
ER+/HER2- & $13(15 \%)$ \\
ER-/HER2- & $2(2 \%)$ \\
ER-/HER2+ & $4(4.5 \%)$ \\
ER+/HER2+ & $40(43 \%)$ \\
Visceral disease & $52(57 \%)$ \\
Non-visceral disease & $40(43 \%)$ \\
Bone only & $29(31.5 \%)$ \\
Initial presentation with metastasis & $10 / 29$ \\
On adjuvant endocrine therapy & $75(81.5 \%)$ \\
No prior chemotherapy & $17(18.5 \%)$ \\
Prior chemotherapy & 11 \\
1 line & 2 \\
2 lines & 2 \\
3 lines & 2 \\
4 lines & 2 \\
Prior trastuzumab & \\
\hline
\end{tabular}

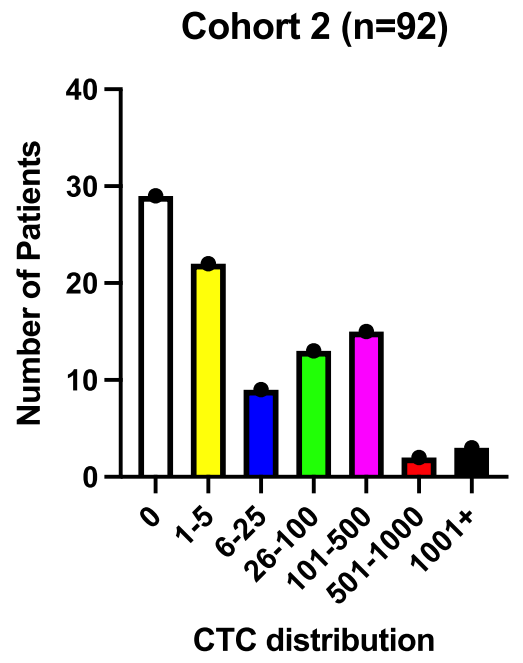

Fig. 3 CTC count distribution cohort 2

\section{Correlation between CTC and OS}

The prognostic significance of baseline CTC count $(<$ or $\geq 5$ CTC $/ 7.5 \mathrm{~mL}$ ) was confirmed in this cohort with a logrank HR of $4.167(p<0.0001)$ (Fig. 4A).

\section{Correlation between In D-dim and OS}

The levels of D-dimer ranged between 100 and 30,000 ng/ $\mathrm{mL}$ with a median of $1125 \mathrm{ng} / \mathrm{mL}$. Increasing levels of fibrin degradation are associated with a shortened OS. $\left(R^{2}=0.252\right.$, $p<0.0001)$. Median OS differed with borderline statistical significance according to the median D-dim (135 days versus 650 days) with a logrank HR of 2.095 (0.917-4.799) $(p=0.068)$.

\section{Correlation between CTC and D-dim level}

Also in this cohort a striking linear correlation is observed between CTC enumeration and D-dimer level $\left(R^{2}=0.3354\right.$, $p<0.0001$ ) (Fig. 4C).

\section{Correlation between CTC and platelet count (PLT)}

The platelet count varied between 43 and $424 \times 10^{9} / \mathrm{mL}$ with a median value of 242 . The PLT level was correlated with the $\ln$ transformed CTC level $\left(p<0.004, R^{2}=0.1902\right)$ (Fig. 4D).

\section{Correlation between D-dim and PLT}

An inverse association between platelet count (PLT) $(10 \%$ $\mathrm{mL})$ and $\ln \mathrm{D}$-dimers $(\mathrm{ng} / \mathrm{mL})$ is also present in this cohort (Fig. 4E) $\left(R^{2}=0.1947, p<0.001\right)$. 
Fig. 4 Cohort 2. A Kaplan-Meier estimates of OS (days) in patients with MBC for those with $<5$ CTCs per $7.5 \mathrm{~mL}$ of whole blood and those in the group with $\geq 5$ CTCs in $7.5 \mathrm{~mL}$ of whole blood $(n=92)$. (HR 4.167, $p<0.0001)$. B Relationship between the ln D-dimers (ng/ $\mathrm{mL})$ versus OS (days) $(n=84)\left(R^{2}=0.252, p<0.0001\right)$. C Relationship between $\ln$ CTC $(x / 7.5 \mathrm{~mL})$ and $\ln$ D-dimers $(\mathrm{ng} / \mathrm{mL})(n=92)$ $\left(R^{2}\right.$ 0.3354, $\left.p<0.0001\right)$. D Relation between platelet count (PLT) $\left(10^{9} / \mathrm{mL}\right)$ and the $\ln$ CTC $(x / 7.5 \mathrm{~mL})(n=92)\left(R^{2} 0.1902, p=0.004\right)$. E Relation between platelet count (PLT) $\left(10^{9} / \mathrm{mL}\right)$ and $\ln$ D-dimers $(\mathrm{ng} / \mathrm{mL})\left(R^{2} 0.1947, p<0.001\right)$

\section{Correlation between extracellular vesicles (ECV) and CTC}

A significant correlation exists between In CTC count and In ECV with a $p<0.001$ and $R^{2}$ of 0.740 . (Fig. 5A). The ECV count is also positively related to the D-dimers in an univariate analysis with $R^{2}=0.4160(p<0.0001)$. In a multiple regression analysis, relating both CTC and ECV with the D-dimers as the dependent variable, the addition of the tdEVs data failed to contribute significantly. Number of tdEVs significantly predicted OS (Fig. 5B).

\section{Univariate and multivariate analysis for OS}

In the univariate analysis, the performance status, visceral disease, number of lines of chemotherapy (0-1 vs 2+), $\mathrm{CTC} \geq 5 \mathrm{CTC} / 7.5 \mathrm{~mL}$, the levels of D-dimers and ECV, serum LDH, HER2 status and ER status were significantly associated with overall survival. Age, platelet count, and fibrinogen level were not.

In multivariate analysis only CTC $\geq 5$ CTC $/ 7.5 \mathrm{~mL}$, ER status, HER2 status and lines of chemotherapy were associated with OS.

\section{Discussion}

The association between cancer and thrombotic events is well established and patients with advanced breast cancer have an increased risk for venous thromboembolic events (VTE). In two cohorts of patients with stage IV breast cancer, and excluding patients with established prior or current TE events and those patients on drugs interfering with normal coagulation, the prevalence of coagulation abnormalities (defined by results crossing the boundaries of the upper limits of normality for either platelet count, D-dimers, fibrinogen, PTT and/or aPTT), was respectively $91 \%$ and $85 \%$ for the retrospective and the prospective cohort. Limiting the analysis to those patients, from both cohorts, presenting with untreated stage IV disease, did not change those frequencies. Nearly all these patients, 35 out of 37 (95\%), had at least one abnormal coagulation test.

The retrospective cohort and the validation cohort both corroborate the prognostic significance of CTC $\geq 5 \mathrm{CTC}$
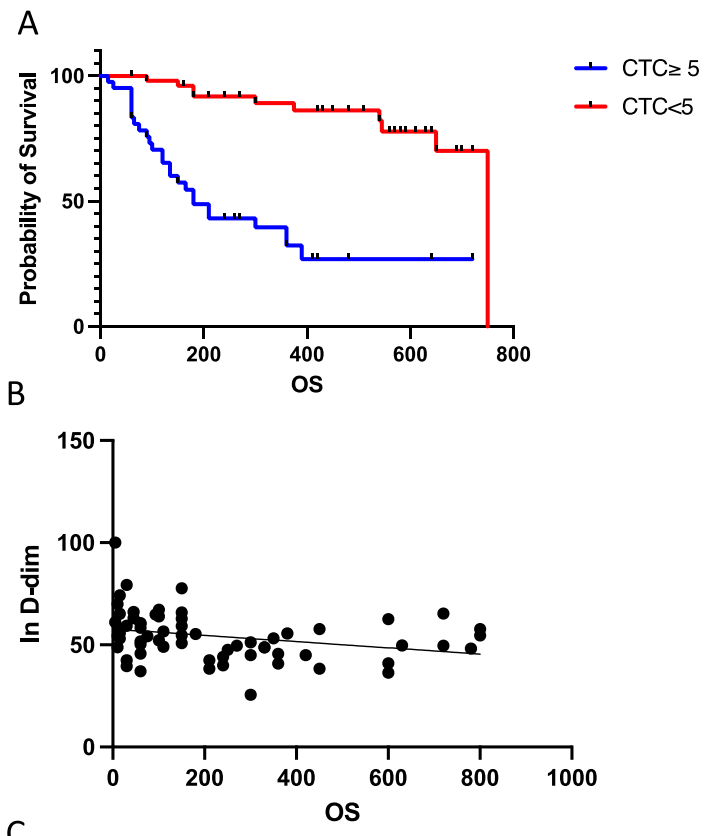

C

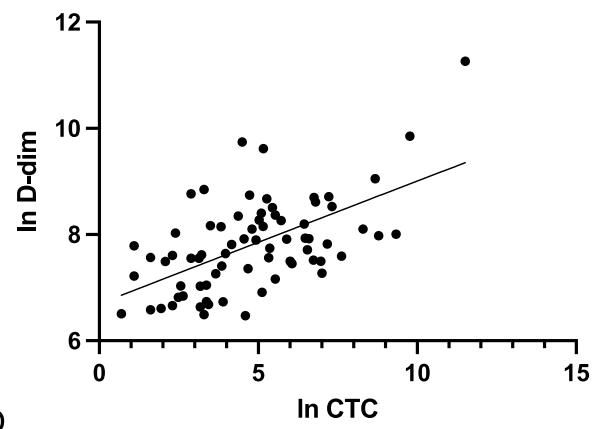

D

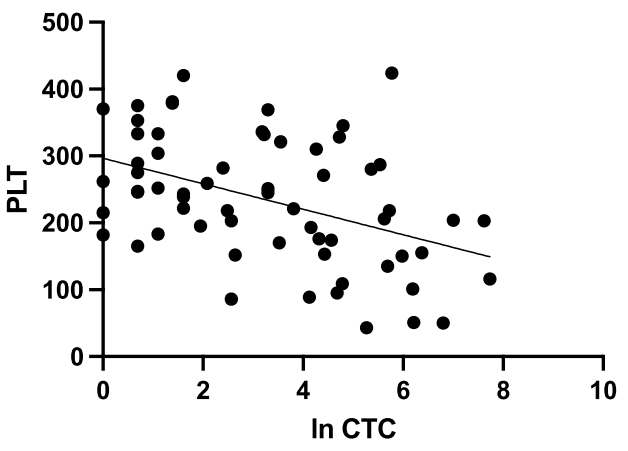

E

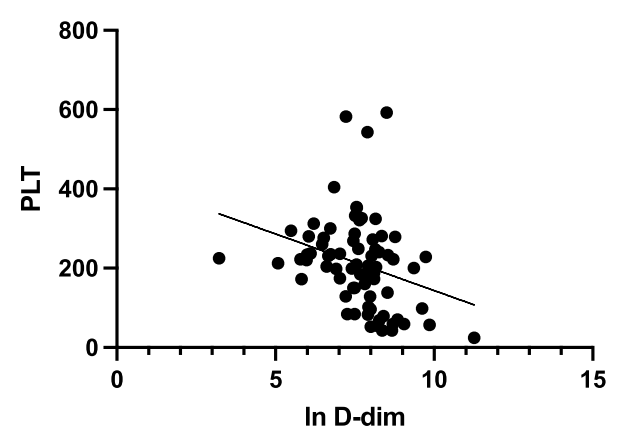


A
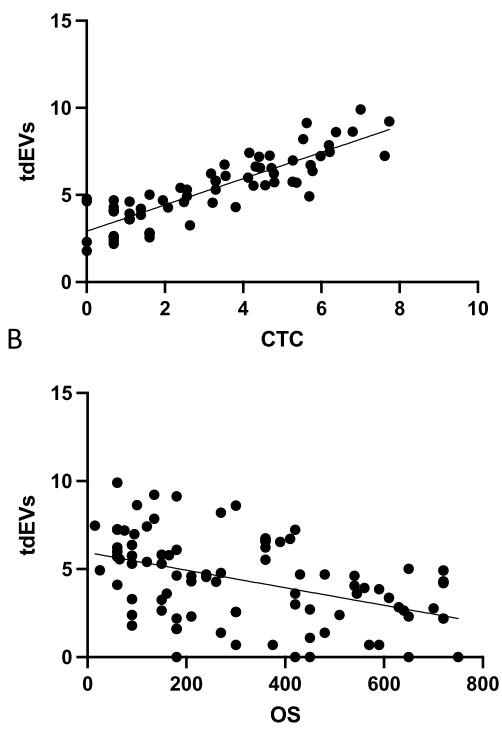

Fig. 5 Cohort 2. A Regression analysis between $\ln$ CTC and $\ln$ tdEVs. $\left(R^{2} 0.743, p<0.0001\right)$ B Relation between $\ln$ tdEVs and OS $(p<0.001)$

$17.5 \mathrm{~mL}$ (Figs. 2A and 4A). The respective HR obtained for OS were $0.35(0.226-0.553)$ and $0.123(0.058-0.256)$ for patients with CTC counts less than 5. Although these two cohorts were enrolled in different time frames, the extent of disease was clearly more advanced in the retrospective cohort. In this cohort 1, 15/77 (19\%) patients had 0 CTC/7.5 mL and 16/77 (20.7\%) had a CTC $<5$. In this cohort 1 , a total $37 / 77(48 \%)$ had a CTC count $>100$ and eleven patients (14\%) had more than 1000 CTCs $/ 7.5 \mathrm{~mL}$, suggesting that in substantial group of patients with endstage disease, extremely elevated numbers of tumor cells are being shed into the circulation. In cohort 2, 29/92 (23\%) patients had 0 CTC/7.5 mL and 47/92 (55.4\%) had a CTC count $<5$. These large differences in fraction of CTC positives and the high absolute numbers of CTCs in some patients in cohort 1 , prompted us to opt for an additional prospectively identified confirmatory cohort of patients with MBC. The degree of CTC positivity in cohort 2 with nearly half of the patients with 5 or more CTC is clearly more in line with what has been reported previously in unselected patients with progressive MBC [25, 26].

In univariate analysis, absolute CTC count was positively associated with D-dimer levels in both cohorts. Similarly, in both cohorts, CTC numbers and D-dimer level were inversely related to platelet count. These relationships were highly significant in both cohorts. Strikingly, the positive correlation between CTC and D-dimer level was the strongest. Others have already observed a correlation between CTC positivity and increased risk for venous thromboembolism [17]. Even a positive categorical association between CTC positivity and D-dimer level has been reported [27, 28]. In both our cohorts, the positive association between CTC and D-dimer, both as a continuous variable, is an important addition to these prior observations. These results corroborate previous studies suggesting that the presence of epithelial cancer cells in the circulation acts directly is an activator of intravascular platelet and coagulation activation [29]. These results are probably an underestimation because of the exclusion of patients with a past or recent thromboembolic event and those patients deemed at a risk for such an event that it warrants drug treatment.

Tumor derived extracellular vesicles as quantified by the ACCEPT program, were significantly associated with CTC count. In univariate analysis EVC number was also associated with coagulation activation. In a multivariate analysis CTC count predominated this association both with D-dimers and platelet, and tdEVs did not add in this association.

In the 10 patients who died within 15 days after blood collection, CTC counts were very high (median count 653 CTCs $/ 7.5 \mathrm{~mL}$, mean 12,000 CTCs $/ 7.5 \mathrm{~mL}$ ), and platelet counts were low (median 72, mean 112) (data not shown). This suggests, accepting all the limitations of low patient numbers and case selection bias, that a "leukemic" phase with consumptive coagulation is more common than generally accepted. We have previously demonstrated in an autopsy study that a substantial fraction of CTCs is retained in the pulmonary vascular bed, and suggested, as others have done previously, that intravascular metastasis is a relevant growth model in patients with advanced disease [30, 31].

In conclusion, our study adds clinical arguments to the hypothesis for a contribution of CTCs as one mechanism of intravascular coagulation activation. We furthermore confirmed the direct association between CTC count and tdEVs. We could not confirm an independent association of these tdEVs with the D-dimer level of platelet count. The role of different types of anticoagulation have been extensively studied in patients with advanced malignancies mainly with the aim of preventing thromboembolic events. Impact of these types of anticoagulation on tumor progression is less clear. Recent studies are investigating a potentially direct antiproliferative effect of direct anticoagulants in patients with operable breast cancer [32]. This study, however lacks any mechanistic/functional analysis of the potential contribution of CTCs in the activation of coagulation. This limits any formal causal conclusion for a link between CTC numbers and coagulation disturbances.

Finally, we suggest that substantial numbers of CTCs are present in terminally ill patients with MBC.

Acknowledgements We would like to thank all patients for participating in the different clinical studies on CTC enumeration and in particular for their part in the $\mathrm{P} 1133$ prospective comparative trial. 
Funding All the authors declare to have no financial or non-financial interests that are directly or indirectly related to the work submitted for publication.

\section{Declarations}

Conflict of interest The authors declare that they have no conflict of interest.

Open Access This article is licensed under a Creative Commons Attribution 4.0 International License, which permits use, sharing, adaptation, distribution and reproduction in any medium or format, as long as you give appropriate credit to the original author(s) and the source, provide a link to the Creative Commons licence, and indicate if changes were made. The images or other third party material in this article are included in the article's Creative Commons licence, unless indicated otherwise in a credit line to the material. If material is not included in the article's Creative Commons licence and your intended use is not permitted by statutory regulation or exceeds the permitted use, you will need to obtain permission directly from the copyright holder. To view a copy of this licence, visit http://creativecommons.org/licenses/by/4.0/.

\section{References}

1. Falanga A, Marchetti M, Vignoli A (2013) Coagulation and cancer: biological clinical aspects. J Thromb Haemost 11:223-233

2. Blackwell K, Haroon Z, Broadwater G, Berry D, Harris L, Iglehart JD, Dewhirst M et al (2000) Plasma D-dimer levels in operable breast cancer patients correlate with clinical stage and axillary lymph node status. J Clin Oncol 18:600-608

3. Mijic S, Dabrosin C (2021) Platelet activation in situ in breasts at high risk of cancer: relationship with mammographic density and estradiol. J Clin Endocrinol Metab 106:485-500

4. Shaker H, Bundred NJ, Landberg G et al (2020) Breast cancer stromal clotting activation (tissue factor and thrombin): a preinvasive phenomena that is prognostic in invasion. Cancer Med 9:1768-1778

5. Mandoj C, Pizzuti L, Sergi D et al (2018) Observational study of coagulation activation in early breast cancer: development of a prognostic model based on data from the real world setting. J Transl Med 16(1):129. https://doi.org/10.1186/ s12967-018-1511-x

6. Dirix LY, Salgado R, Weytjens R, Colpaert C, Benoy I, Huget P et al (2002) Plasma fibrin D-dimer levels correlate with tumor volume, progression rate and survival in patients with metastatic breast cancer. Brit J Cancer 86:389-395

7. Lu Y, Zhang L, Zhang Q, Zhang Y, Chen D, Lou J, Jiang J, Ren C (2019) PLoS ONE 14:e0221374

8. Nagy JA, Brown LF, Senger DR, Lanir N, Van de Water L, Dvorak AM, Dvorak HF (1989) Pathogenesis of tumor stroma generation: a critical role for leaky blood vessels and fibrin deposition. Biochim Biophys Acta 948(3):305-26

9. Dvorak HF (1986) Tumors: wounds that do not heal. Similarities between tumor stroma generation and wound healing. N Engl J Med 315(26): 1650-1659

10. Shoji M, Hancock WW, Abe K, Micko C, Casper KA, Baine RM et al (1998) Activation of coagulation and angiogenesis in cancer: immunohistochemical localization in situ of clotting proteins and vascular endothelial growth factor in human cancer. Am J Pathol 152:399-411
11. Dirix LY, Vermeulen PB, Pawinski A, Prové A, Benoy I, De Pooter C, Martin M, Van Oosterom AT (1997) Elevated levels of the angiogenic cytokines basic fibroblast growth factor and vascular endothelial growth factor in sera of cancer patients. Br J Cancer 76(2):238-243

12. Vermeulen PB, Salven P, Benoy I, Gasparini G, Dirix LY (1999) Blood platelets and serum VEGF in cancer patients. Br J Cancer 79(2):370-373

13. Salgado R, Benoy I, van Marck E, Vermeulen P, Dirix L (2002) The importance of the VEGF-load in platelets in cancer patients. Ann Oncol 13(10):1689-1690

14. Feinauer MJ, Schneider SW, SW, Berghoff AS, et al (2021) Local blood coagulation drives cancer cell arrest and brain metastasis in a mouse model. Blood 137(9):1219-1232. https://doi.org/10. 1182/blood.2020005710

15. Lucotti S, Muschel RJ (2020) Platelets and Metastasis: new Implications of an old Interplay. Front Oncol 10:1350. https:// doi.org/10.3389/fonc.2020.01350.eCollection

16. Ren J, He J, Zhang H, Xia Y et al (2021) Platelet TLR4ERK5 axis facilitates NET-mediated capturing of circulating tumor cells and distant metastasis after surgical stress. Cancer Res 81(9):2373-2385. https://doi.org/10.1158/0008-5472. CAN-20-3222

17. Mego M, De Giorgi U, Broglio K, Dawood S, Valero V, Andreopoulou E et al (2009) Circulating tumour cells are associated with increased risk of venous thromboembolism in metastatic breast cancer patients. Br J Cancer 101(11):1813-1816

18. Chew HK, Wun T, Harvey DJ, Zhou H, White RW (2007) Incidence of venous thromboembolism and the impact on survival in breast cancer patients. J Clin Oncol 25(1):70-76

19. Levi M (2019) Disseminated intravascular coagulation in cancer: an update. Semin Thromb Hemost 45:342-347

20. Salagado R, Benoy I, Weytjens R, Van Bockstaele D, Van Marck E, Huget $P$ et al (2002) Arterio-venous gradients of IL-6, plasma and serum VEGF and D-dimers in human cancer. Brit J Cancer 87:1437-1444

21. Yohei Hisada Y, Nigel Mackman N (2021) Tissue factor and extracellular vesicles: activation of coagulation and impact on survival in cancer. Cancers 13(15):3839

22. Van Der Auwera I, Peeters D, Benoy IH, Elst HJ, Van Laere SJ, Prové A et al (2010) Circulating tumour cell detection: a direct comparison between the cell search system, the AdnaTest and CK-19/mammaglobin RT-PCR in patients with metastatic breast cancer. Br J Cancer 102:276-284

23. Peeters DJE, van Dam P-J, Van den Eynden GGM, Rutten A, Wuyts H, Pouillon L et al (2014) Detection and prognostic significance of circulating tumour cells in patients with metastatic breast cancer according to immunohistochemical subtypes. Br J Cancer 110:375-383

24. Kraan J, Sleijfer S, Strijbos MH, Ignatiadis M, Peeters D, Pierga J-Y et al (2011) External quality assurance of circulating tumor cell enumeration using the Cell Search $(\circledR)$ system: a feasibility study. Cytometry 80:112-118

25. Bidard F-C, Peeters DJ, Fehm T, Nolè F, Gisbert-Criado R, Mavroudis D et al (2014) Clinical validity of circulating tumour cells in patients with metastatic breast cancer: a pooled analysis of individual patient data. Lancet Oncol 15:406-414

26. Nanou A, Miller MC, Zeune LL, de Wit S, Punt CJA, Groen HJM, Hayes DF, de Bono JS, Terstappen LWMM (2020) Tumour-deriverd extracellular vesicles in blood of metastatic cancer patients associate with overall survival. Brit J Cancer 122:801-811

27. Mego M, Karaba M, Minarik G, Benca J, Sedlackova T, Tothova L et al (2015) Relationship between circulating tumor cells, blood coagulation, and urokinase-plasminogen-activator system in early breast cancer. Breast J 21(2):155-160 
28. Kirwan CC, Descamps T, Castle J (2020) Circulating tumour cells and hypercoagulability: a lethal relationship in metastatic breast cancer. Clin Transl Onc 22:870-877

29. Bystricky B, Reuben JM, Mego M (2017) Circulating tumor cells and coagulation-minireview. Crit Rev Oncol Hematol 114:33-42

30. Peeters DJ, Brouwer A, Van den Eynden G et al (2015) Circulating tumour cells and lung microvascular tumour cell retention in patients with metastatic breast and cervical cancer. Cancer Lett 356:872-879

31. Al-Mehdi AB, Tozawa K, Fisher AB, Shientag L, Lee A, Muschel $\mathrm{RJ}$ (2000) Intravascular origin of metastasis from the proliferation of endothelium-attached tumor cells: a new model for metastasis. Nat Med 6(1):100-102
32. Castle J, Blower E, Bundred NJ, Harvey JR, Thachil J, Marshall A et al (2020) Rivaroxaban compared to no treatment in ER-negative stage I-III early breast cancer patients (the TIP-trial): study protocol for a phase II preoperative window-of-opportunity study design randomized controlled trial. Trials 21:749

Publisher's Note Springer Nature remains neutral with regard to jurisdictional claims in published maps and institutional affiliations. 